\title{
Differentiating Between Cancer and Inflammation: \\ A Metabolic-Based Method for Functional Computed Tomography Imaging
}

Menachem Motiei ${ }^{1+}$, Tamar Dreifuss ${ }^{1+}$, Oshra Betzer ${ }^{1}$, Hana Panet $^{1}$, Aron Popovtzer ${ }^{2}$, Jordan Santana ${ }^{1}$, Galith Abourbeh ${ }^{3}$, Eyal Mishani ${ }^{3}$ and Rachela Popovtzer ${ }^{1 *}$

${ }^{1}$ Faculty of Engineering and the Institutes of Nanotechnology \& Advanced Materials, Bar-llan University, Ramat-Gan, Israel. ${ }^{2}$ Davidoff cancer center, Rabin Medical Center, Beilinson Campus, Petah Tiqwa, Israel. ${ }^{3}$ Cyclotron-Radiochemistry-MicroPET Unit, Hadassah-Hebrew University Hospital, Jerusalem,
91120 , Israel.

Correspondence should be addressed to R.P. (email: rachela.popovtzer@biu.ac.il)

${ }^{+}$These authors contributed equally to this work 


\section{Supplementary information}

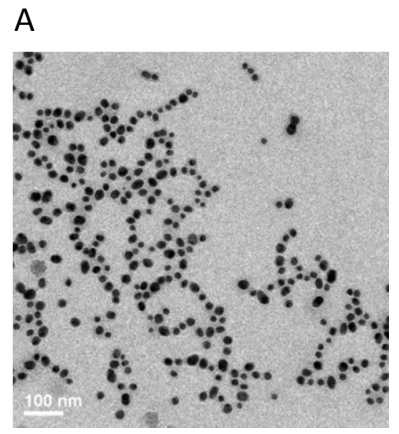

B

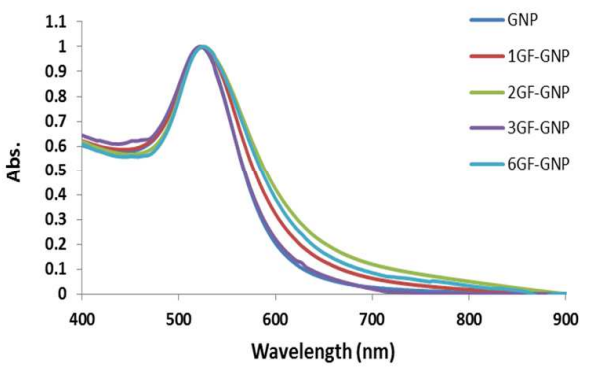

C

\begin{tabular}{|c|c|c|}
\hline & $\begin{array}{c}\text { Zeta } \\
\text { potential } \\
(\mathrm{mV})\end{array}$ & $\begin{array}{c}\text { Size } \\
(\mathbf{d} . \mathrm{nm})\end{array}$ \\
\hline Bare GNP & $-37.1( \pm 1.8)$ & $30.1( \pm 1.3)$ \\
\hline PEG7-GNP & $-42.3( \pm 2.2)$ & $35.2( \pm 0.3)$ \\
\hline 1GF-GNP & $-17.7( \pm 2.6)$ & $51.4( \pm 2.1)$ \\
\hline 2GF-GNP & $-15.9( \pm 0.9)$ & $49.6( \pm 2.2)$ \\
\hline 3GF-GNP & $-15.0( \pm 1.1)$ & $46.5( \pm 2.0)$ \\
\hline 6GF-GNP & $-16.2( \pm 1.6)$ & $45.9( \pm 1.7)$ \\
\hline
\end{tabular}

Figure S1: Characterization of GF-GNPs: A) TEM image of $20 \mathrm{~nm}$ GNPs (scale bar $100 \mathrm{~nm}$ ). B) Optical properties of GNPs: ultraviolet visible spectroscopy of bare GNPs and 4 types of glucose-coated GNPs, differing in the intra-molecular glucose site being functionalized: 1GF-GNP, 2GF-GNP, 3GF-GNP and 6GF-GNP. C) Zeta potential and DLS measurements (hydrodynamic diameter) of the various GNPs. The differences obtained by UV-vis spectroscopy, zeta potential and DLS following each conjugation step demonstrate the efficiency of the chemical coating. In addition, the similarity between the properties of the four types of GF-GNPs indicates the same physicochemical characteristics. 

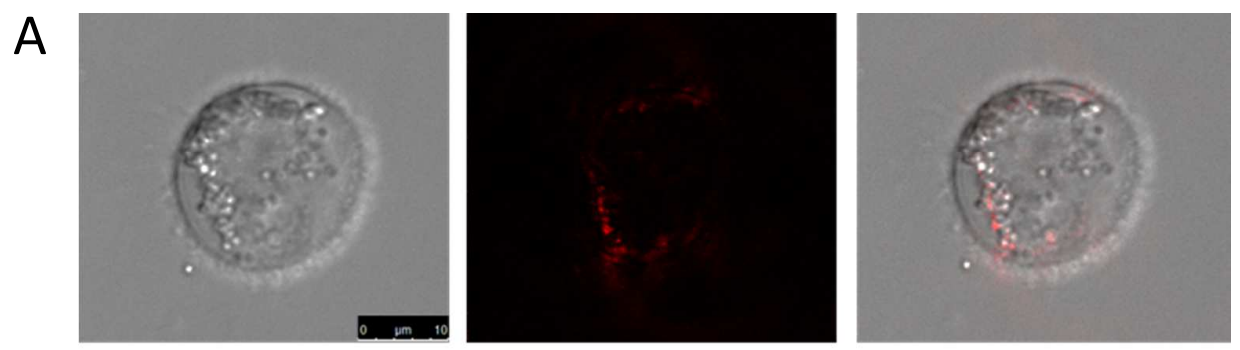

B
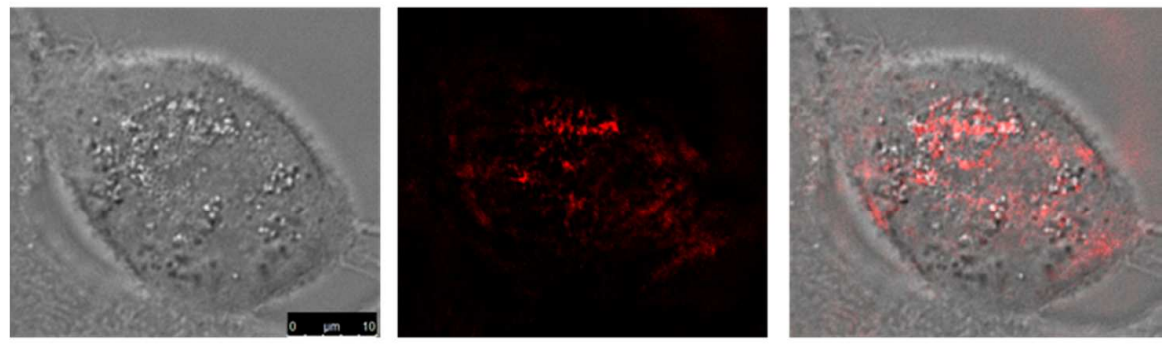

C
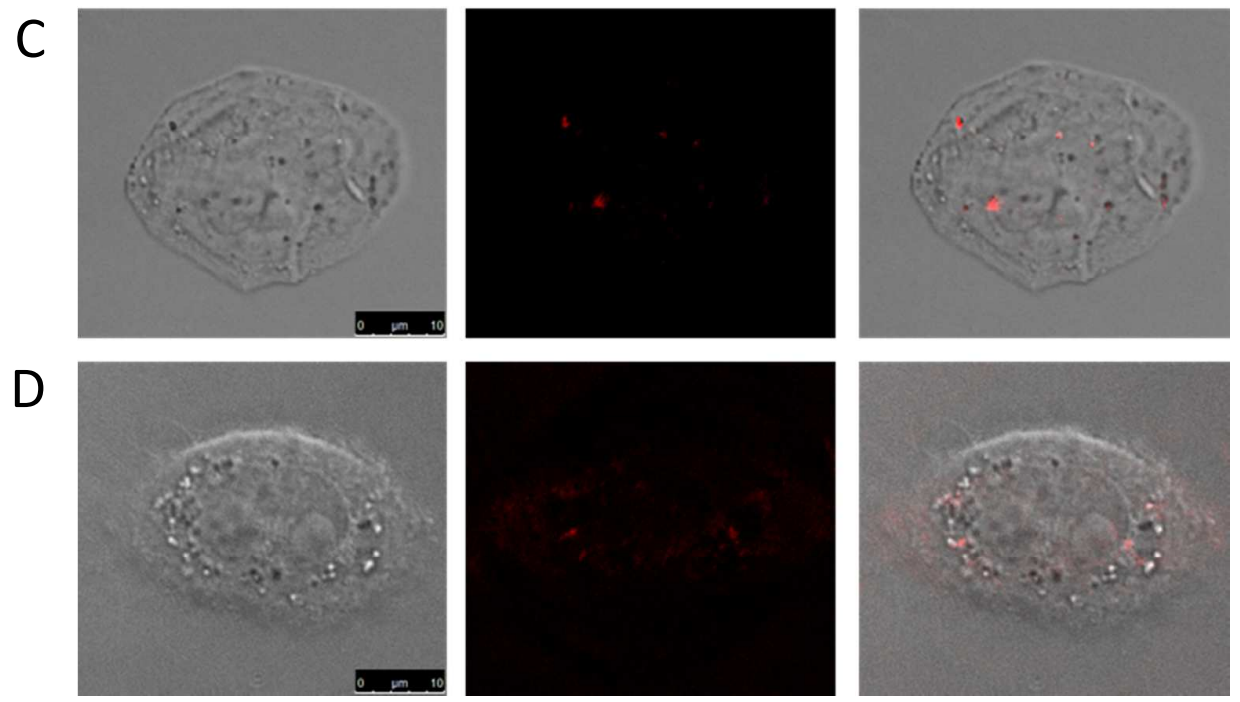

Figure S2: Confocal images of A431 cells after 30 min incubation with: A) Rhodamine B-1GF-GNP complex. B) Rhodamine B-2GF-GNP complex. C) Rhodamine B-3GF-GNP complex and D) Rhodamine B-6GF-GNP complex. Left: bright field image of the cell; middle: fluorescent-coated 2GF-GNPs (red); right: combined images. 


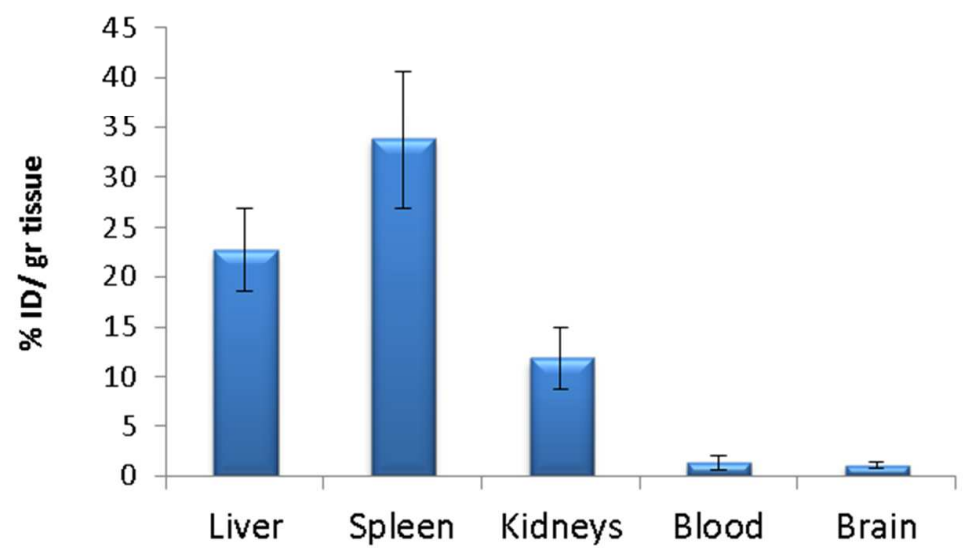

Figure S3: Biodistribution of 2GF-GNP in the main organs at $3.5 \mathrm{~h}$ post injection. Results presented as mean \pm SEM. 
A

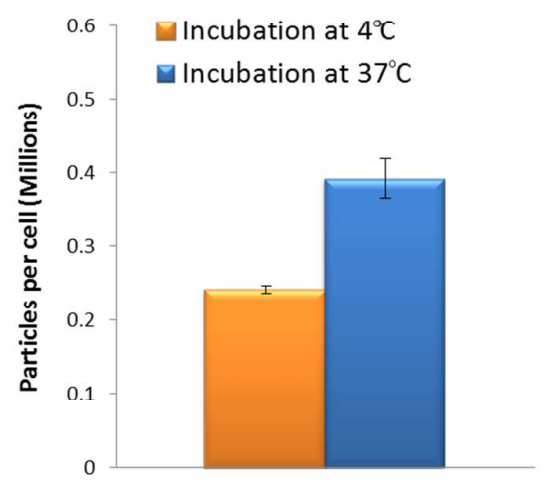

B

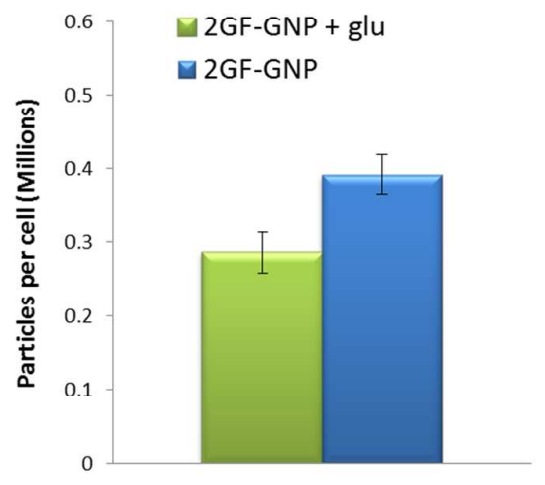

C

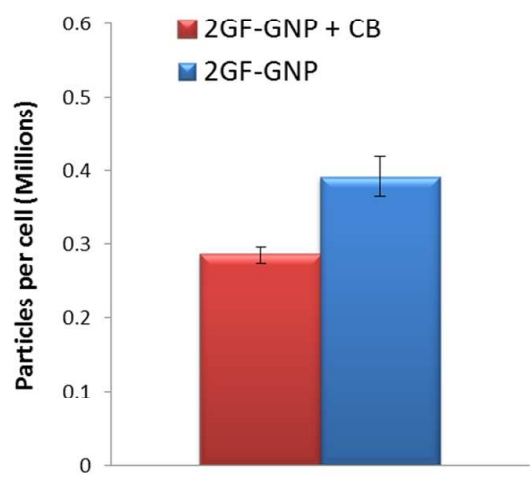

Figure S4: Internalization mechanism study in 3 T3 cell line. A) 2GF-GNPs internalization after 30 min incubation at $37^{\circ} \mathrm{C}$ in comparison to $4^{\circ} \mathrm{C}$. 2GF-GNPs were internalized to a lesser extent when kept at $4^{\circ} \mathrm{C}$ compared to $37^{\circ} \mathrm{C}$. B) Saturation with free glucose: 2GF-GNP uptake after 30 min incubation with high glucose medium $(10 \mathrm{mg} / \mathrm{mL}$ ) was lower than after incubation with normal glucose levels $(4.5 \mathrm{mg} / \mathrm{mL})$. C) Cytochalasin $B$ inhibition test: pre-incubation with $\mathrm{CB}$ has significantly inhibited the uptake of 2GF-GNP. Results presented as mean $\pm S D$. 